\title{
Preferência Manual na Ação de Alcançar em Bebês em Função da Localização Espacial do Alvo
}

\author{
Infants' Manual Preference in Reaching Regarding Spatial Target Location
}

\author{
Rosana Machado Souza ${ }^{*}, a, b$, Eloisa Tudella ${ }^{a}, \&$ Luis Augusto Teixeira ${ }^{b}$ \\ ${ }^{a}$ Universidade Federal de São Carlos, São Carlos, Brasil \& ${ }^{b}$ Universidade de São Paulo, São Paulo, Brasil
}

\begin{abstract}
Resumo
Preferência manual em bebês de 5 meses de idade foi avaliada em tarefas de alcançar alvos estáticos posicionados na linha média, à direita e à esquerda com referência a coordenadas egocêntricas. A análise da frequência de uso das mãos indicou direção e magnitude de preferência manual diversificadas entre os bebês. Foi observado que os bebês realizaram predominantemente alcances ipsilaterais para alvos posicionados lateralmente. Para alcances na linha média a frequência de uso foi semelhante entre as mãos direita e esquerda. Os resultados sugerem que nessa fase do desenvolvimento a preferência manual para o alcançar é alterada pela posição dos objetos no espaço.

Palavras-chave: Lateralidade; Preferência manual; Desenvolvimento motor; Bebês.
\end{abstract}

\begin{abstract}
Manual preference of 5-month-old infants was evaluated in tasks of reaching static targets positioned on the midline, at the left and right, regarding egocentric coordinates. Frequency analysis of using the hands pointed out to diversified direction and magnitude of manual preference among infants. It was observed that they predominantly made ipsilateral reaches toward targets laterally positioned. Frequency of using the right and left hands was similar for targets located at the midline. The results suggest that in this stage of development manual preference is shifted by the spatial position of the objects.

Keywords: Laterality; Manual preference; Motor development; Infants.
\end{abstract}

O uso preferencial de uma mão em relação ao uso da mão oposta é uma característica comum nos seres humanos. Aproximadamente $85-90 \%$ de indivíduos adultos têm relatado possuir preferência manual direita (Brackenridge, 1981; Porac, Coren, \& Duncan, 1980). Evidências indicam que a lateralização do comportamento é iniciada precocemente. Ramsay (1980), ao avaliar a preferência manual de bebês aos 5, 7, 9 e 13 meses, não encontrou preferência manual em bebês aos 5 meses. Entre 7 e 9 meses de idade a maioria dos bebês mostrou preferência manual, a qual foi confirmada aos 13 meses, sugerindo que o estabelecimento da preferência manual se inicia na segunda metade do primeiro ano de vida do bebê. Caplan e Kinsbourne (1976), no entanto, detectaram preferência manual em bebês em um período anterior ao observado por Ramsay, quando os bebês possuíam apenas 3 meses

\footnotetext{
* Endereço para correspondência: Laboratório Sistemas Motores Humanos, Escola de Educação Física e Esporte, USP, SP. CEP 05508-030. Tel.: (11) 3091-8795. E-mails: romasouza@yahoo.com.br, eloisatudella@yahoo.com e lateixeissmh@hotmail.com

Os autores agradecem à Fundação de Amparo à Pesquisa do Estado de São Paulo (FAPESP) pela bolsa de estudos concedida ao primeiro autor (\#2008/02145-8) e ao ConselhoNacional de Desenvolvimento Científico e Tecnológico $(\mathrm{CNPq})$ pela bolsa produtividade concedida ao terceiro (\#308312/2006-6) autor.
}

de idade. As assimetrias laterais mais precoces foram detectadas em períodos pré-natais (Hepper, McCartney, \& Shannon, 1998; Hepper, Shahidullah, \& White, 1991). Hepper et al. (1998) observaram que 85\% dos fetos com apenas 10 semanas de idade gestacional exibiram mais movimentos com o braço direito do que com o braço esquerdo, revelando indícios das primeiras manifestações da lateralidade.

Apesar da precocidade das primeiras manifestações de comportamento lateralizado, estudos prévios têm indicado que os primeiros anos de vida são marcados por alternâncias na preferência manual (Corbetta \& Thelen, 1996; Gesell \& Ames, 1947). Tais alternâncias têm sido concebidas como derivadas de experiências sensóriomotoras ao longo do processo de desenvolvimento. Isto é, o desenvolvimento de habilidades motoras fundamentais, tais como sentar independente, engatinhar e andar, parecem estar associadas a mudanças na preferência lateral observadas no primeiro ano de vida (Corbetta \& Thelen, 1999; Corbetta, Williams, \& Snapp-Childs, 2006; Hopkins \& Rönnqvist, 2002). Uma evidência que reforça esse ponto de vista foi oferecida em um estudo com dois bebês que desenvolveram padrões incomuns nas habilidades de arrastar/engatinhar, durante o primeiro ano de vida (Corbetta et al., 2006). Um deles engatinhava movimentando ambos os braços e as pernas simetrica- 
mente. O outro bebê desenvolveu uma forma assimétrica de locomoção, através da qual se locomovia arrastando-se sobre o abdômen e impulsionando o corpo com o braço esquerdo, mantendo o braço direito livre para alcançar e agarrar objetos ao seu redor. Ambos os bebês foram avaliados em uma tarefa de alcance e em relação às habilidades posturais e locomotoras por um período de 17 semanas. Nas primeiras avaliações do movimento de alcance, ambos os bebês apresentaram alcance unimanual com preferência manual esquerda. Porém, concomitantemente ao desenvolvimento da locomoção, desenvolveram preferência manual correspondente ao padrão de movimento usado para se locomover. O primeiro bebê desenvolveu o alcance bimanual, enquanto o segundo passou a apresentar preferência manual direita. Esses achados sugerem uma relação entre as mudanças na preferência manual no primeiro ano de vida e a prática em novas experiências sensório-motoras. Nesse sentido, fica aparente que em determinados períodos do ciclo de vida elementos ambientais, tais como experiências sensoriais e motoras, podem desempenhar um papel mais importante no desenvolvimento da lateralidade do que fatores biológicos.

A noção de formação da preferência manual a partir da interação com o ambiente tem sido investigada em uma série de estudos enfocando outros aspectos dessa interação. Achados de pesquisas em bebês indicam congruência entre a preferência manual do bebê e a preferência manual da mãe, enquanto que o mesmo não tem sido verificado em relação à preferência manual do pai (cf. Ashton, 1982; Harkins \& Michel, 1988). Em famílias com pais discordantes em relação à preferência manual, a descendência manual esquerda provém com maior frequência da mãe do que do pai (Annett, 1978, 1999). Harkins e Uzgiris (1991) sugerem que a influência materna na preferência manual do bebê pode estar relacionada à interação social entre a mãe e o bebê por comportamento imitativo, mais do que à herança genética. A interação social também parece desempenhar um importante papel na formação da preferência manual em diferentes culturas, nas quais fica evidente a pressão social contra o uso da mão esquerda (cf. Meng, 2007; Singh, Manjary, \& Dellatolas, 2001; Zverev, 2006). Além desses aspectos relacionados à interação com o ambiente, resultados de pesquisas têm indicado que indivíduos do sexo feminino apresentam maior tendência à preferência manual direita (Annett, 1970; Sacco, Moutard, \& Fagard, 2006). Esse fato tem sido atribuído a fatores genéticos e hormonais (Strogonova, Pushina, Orekhova, Posikera, \& Tsetlin, 2004).

Uma evidência adicional da influência do ambiente no desenvolvimento da lateralidade infantil é de que a disposição espacial, em coordenadas egocêntricas, de objetos com os quais o bebê interage tem sido demonstrada ser um elemento relevante na indução de preferências laterais. Morange e Bloch (1996) realizaram um estudo com bebês de 4 a 7 meses de idade com o objetivo de avaliar preferências laterais durante o alcance e preensão de objetos colocados em três posições: linha média, lado esquerdo e lado direito, em relação ao plano sagital mediano do corpo do bebê. Os resultados indicaram que alcances dos objetos localizados lateralmente foram sempre ipsilaterais, enquanto que objetos posicionados na linha média foram alcançados mais frequentemente com a mão esquerda (ver Rönnqvist \& Domellöf, 2006, para mesma tendência). Uso mais frequente da mão esquerda tem sido observado também para alcançar objetos posicionados ipsilateralmente em estudos em crianças entre 5 e 8 anos de idade (Leconte \& Fagard, 2006) e adultos (Bryden \& Roy, 2006). Esses dados indicam que a disposição de objetos, com referência a coordenadas egocêntricas, influi na escolha da mão usada no alcance.

Apesar de estudos prévios terem investigado a preferência manual de indivíduos adultos, crianças e bebês, a maior parte deles tem enfocado a direção da preferência manual, não investigando sua magnitude. Em um estudo com estudantes universitários, Peters e Murphy (1992) investigaram a preferência manual a partir de um inventário contendo itens relacionados à realização de tarefas unimanuais. Foram detectadas cinco categorias de preferência manual: consistente esquerda, fraca esquerda, inconsistente esquerda, fraca direita e consistente direita. Os resultados revelaram que entre indivíduos com preferência manual direita a maioria foi consistente, enquanto que entre indivíduos com preferência manual esquerda a distribuição entre as categorias de preferência foi similar. Esses dados levam à conclusão de que, apesar de alguns indivíduos demonstrarem a mesma direção da preferência manual - direita ou esquerda -, a classificação em diferentes categorias permite a identificação de perfis distintos de preferência manual entre os indivíduos adultos. Tal questão ainda não foi investigada em bebês e corresponde a uma lacuna importante no conhecimento sobre o início do processo de lateralização do comportamento humano.

O objetivo do presente estudo foi analisar a direção e magnitude da preferência manual em bebês de 5 meses de idade em tarefas de alcançar objetos estáticos dispostos em diferentes posições em relação a coordenadas egocêntricas ${ }^{1}$. Além disso, foram verificadas a influência do sexo sobre a preferência manual e a congruência entre a preferência manual do bebê e dos seus pais. A apresentação do brinquedo em diferentes posições espaciais permitiu uma análise mais detalhada da preferência manual, indicando a extensão em que pequenas alterações espaciais na posição do alvo induzem a escolha do membro para realizar a ação de alcançar. $\mathrm{O}$ aspecto original da presente investigação foi realizar uma análise mais refinada da preferência manual, discriminando não apenas a direção, mas também a magnitude da preferência ma-

O termo "coordenadas egocêntricas" corresponde à localização de objetos no espaço de trabalho do bebê em relação ao plano sagital mediano, com divisão em hemicampo direito e esquerdo. 
nual. Este aspecto do estudo teve como propósito revelar se as diferentes categorias de manualidade observadas em indivíduos adultos (Peters \& Murphy, 1992) são detectáveis em estágios iniciais do desenvolvimento da preferência manual. A hipótese aqui formulada é de que o posicionamento do brinquedo influi na escolha da mão para alcançá-lo, induzindo alcances ipsilaterais aos alvos laterais e distribuição mais simétrica da preferência manual na posição central. Mais especificamente, se a diversidade de categorias de preferência manual, como observado em indivíduos adultos, for detectável nos primeiros meses de vida dos bebês, deverá ser observada uma variação do perfil de preferência manual em função da posição do alvo no espaço.

\section{Método}

\section{Participantes}

Participaram deste estudo 22 bebês com 5 meses de idade ( $M=5$ meses e 7 dias, $D P=4$ dias), sendo 9 do sexo feminino e 13 do sexo masculino. Foram incluídos no estudo bebês a termo, com idade gestacional de 37 a 41 semanas ( $M=38,6$ semanas, $D P=1,3$ semanas), índice de Apgar igual ou superior a 8 no primeiro $(M=8,8, D P=0,6)$ e quinto $(M=9,8, D P=0,4)$ minutos, ausência de complicações durante o parto ou relato de qualquer outra alteração que pudesse trazer prejuízo ao desenvolvimento neuromotor, cognitivo e afetivo do bebê. Os dados da gestação e do nascimento e as informações relacionadas às condições clínicas do bebê foram obtidos a partir de consulta à Caderneta da Criança, a qual é fornecida para cada bebê imediatamente após o nascimento. Para a admissão dos bebês no estudo, seus pais assinaram um termo de consentimento livre e esclarecido. Este estudo foi aprovado pelo Comitê de Ética local (parecer $\mathrm{n}^{\circ}$. 264/2008).

\section{Materiais e Procedimentos}

Para início da avaliação, os bebês foram posicionados em uma cadeira infantil reclinada em $50^{\circ}$ em relação ao plano horizontal (mesma posição empregada por Von Hofsten, 1982). Foram utilizados três brinquedos coloridos e pequenos (aproximadamente $6 \mathrm{~cm}$ de diâmetro), com formato e peso similares, para eliciar o alcance. Os brinquedos eram posicionados na linha média, à direita e à esquerda em relação ao eixo sagital mediano do corpo do bebê. As posições laterais foram orientadas em função da linha dos ombros dos bebês (como usado anteriormente por Rönnqvist \& Domellöf, 2006), correspondendo a uma distância lateral de aproximadamente $7 \mathrm{~cm}$ em relação ao plano sagital mediano. Os brinquedos eram apresentados a uma distância do bebê correspondente ao comprimento entre o seu ombro e punho, aproximadamente $20-25 \mathrm{~cm}$. A exposição do brinquedo era mantida por um período aproximado de até 60s. Nos casos em que o bebê não realizava o alcance neste período, o brinquedo original era substituído por outro com características semelhan- tes. Uma haste, localizada em frente à cadeira, foi usada como referência para manter fixa a posição de apresentação do brinquedo. A sequência das posições de apresentação do brinquedo foi igual para todos os bebês. Inicialmente, o brinquedo era apresentado na linha média, seguido pelas apresentações à direita e finalmente à esquerda. Os bebês realizavam cinco tentativas de alcance em cada uma das três posições.

Após cada alcance, era permitido que o bebê manipulasse o brinquedo por alguns instantes. Em seguida, o brinquedo era retirado do campo visual do bebê e reapresentado para a tentativa seguinte. Este procedimento era repetido até que o bebê realizasse cinco tentativas de alcance para cada posição de apresentação do brinquedo. O experimentador apresentava os brinquedos ao bebê com as duas mãos, para prevenir que a mão usada pelo experimentador influenciasse a escolha da mão pelo bebê (cf. Fagard \& Lemoine, 2006; Nagy et al., 2005).

Para registro da avaliação, foram utilizadas três câmeras de vídeo digitais, sendo uma da marca JVC (modelo GY DV-300) e duas da marca Sony (DSR-PD170), acopladas a tripés. Uma câmera foi posicionada póstero-superiormente à cadeira infantil, à altura de $1,93 \mathrm{~m}$, e as outras duas localizadas lateralmente à cadeira, estando uma à direita e outra à esquerda, à distância de $1,18 \mathrm{~m}$, e altura de $1 \mathrm{~m}$ (adaptado de Rocha, Silva, \& Tudella, 2006). Os movimentos dos bebês foram registrados em fitas de vídeo digitais e, posteriormente, as imagens foram transferidas para um computador. Para a análise das imagens foi empregado o Sistema DVideo 5.0 (Barros, Brenzikofer, Leite, \& Figueroa, 1999), o qual permitiu a verificação e classificação de cada movimento de alcance com exatidão. A avaliação da preferência manual dos pais foi realizada por meio do Inventário de Dominância Lateral de Edimburgo (Oldfield, 1971).

\section{Análise da Preferência Manual}

A preferência manual foi analisada em função da frequência de alcances com cada mão, que consistiu no número de vezes que o bebê realizou o alcance com as mãos direita e esquerda em cada posição de apresentação do brinquedo. Um alcance foi considerado válido quando o bebê localizava o objeto no espaço e realizava o movimento com um ou ambos os membros superiores em direção ao alvo até tocá-lo (Thelen, Corbetta, \& Spencer, 1996). Nos casos em que apenas uma das mãos tocava o brinquedo o alcance foi classificado como unimanual. $\mathrm{O}$ alcance foi classificado como bimanual quando as duas mãos tocavam o objeto após ambas terem sido dirigidas simultaneamente ao alvo durante pelo menos um quarto da trajetória do alcance, independentemente da ação ter sido iniciada com uma ou com as duas mãos. Nesta classificação não foi considerado o atraso entre as mãos no início do alcance nem no momento do toque no brinquedo (cf. Fagard, 2000).

A preferência manual em cada posição do brinquedo foi estimada por meio do índice de preferência manual, 
dado pela seguinte equação: $I P=(D-E) /(D+E)$, na qual $D$ e E referem-se à frequência absoluta de alcances realizados com as mãos direita e esquerda, respectivamente. Para alcances unimanuais foi atribuído um ponto para a mão que tocou o objeto. Nos casos de alcance bimanual foi atribuído um ponto para cada mão. A força da preferência manual foi determinada através da média entre os índices observados em cada posição de apresentação do brinquedo. Portanto, quanto mais próximo de 1 , maior a preferência pela mão direita; quanto mais próximo de -1 , maior a preferência pela mão esquerda. Índices entre - 0,2 e 0,2 foram considerados como preferência manual indefinida. Assim, em relação à magnitude a preferência manual foi classificada nas seguintes categorias: forte preferência manual esquerda $(\mathrm{FE})$, quando o índice ficou entre - 1 e $-0,81$; moderada esquerda (ME), quando o índice ficou entre $-0,8$ e $-0,51$; fraca esquerda (FrE), quando o índice ficou entre $-0,5$ e $-0,21$; indefinida (IND), quando o índice ficou entre $-0,2$ e 0,2 ; fraca direita (FrD), quando o índice ficou entre 0,21 e 0,5 ; moderada direita (MD), quando o índice ficou entre 0,51 e 0,8 ; forte direita (FD), quando o índice ficou entre 0,81 e 1.

Para testar o efeito da interação entre as posições de apresentação do brinquedo e o sexo sobre a preferência manual foi empregado um modelo de análise de variância de dois fatores, 2 (sexo) x 3 (posição: linha média x direita $x$ esquerda), com medidas repetidas no segundo fator. A prova de Newman-Keuls foi empregada para realizar as comparações discriminantes. O nível mínimo de significância foi estabelecido em $5 \%$.

\section{Resultados}

A análise do índice de preferência manual considerando todas as posições do brinquedo indicou que nove bebês apresentaram preferência manual direita, nove apresentaram preferência manual esquerda e quatro apresentaram preferência manual indefinida. Na Figura 1 é apresentada a frequência absoluta de bebês classificados nas categorias estabelecidas. Interessante notar que foram identificadas as mesmas frequências de bebês com preferência manual direita e com preferência manual esquerda. Outro aspecto de interesse foi a distribuição diversificada das preferências manuais, com variação entre forte preferência esquerda e forte preferência direita. A maioria dos bebês se concentrou nas categorias de fraca preferência manual e indefinida.

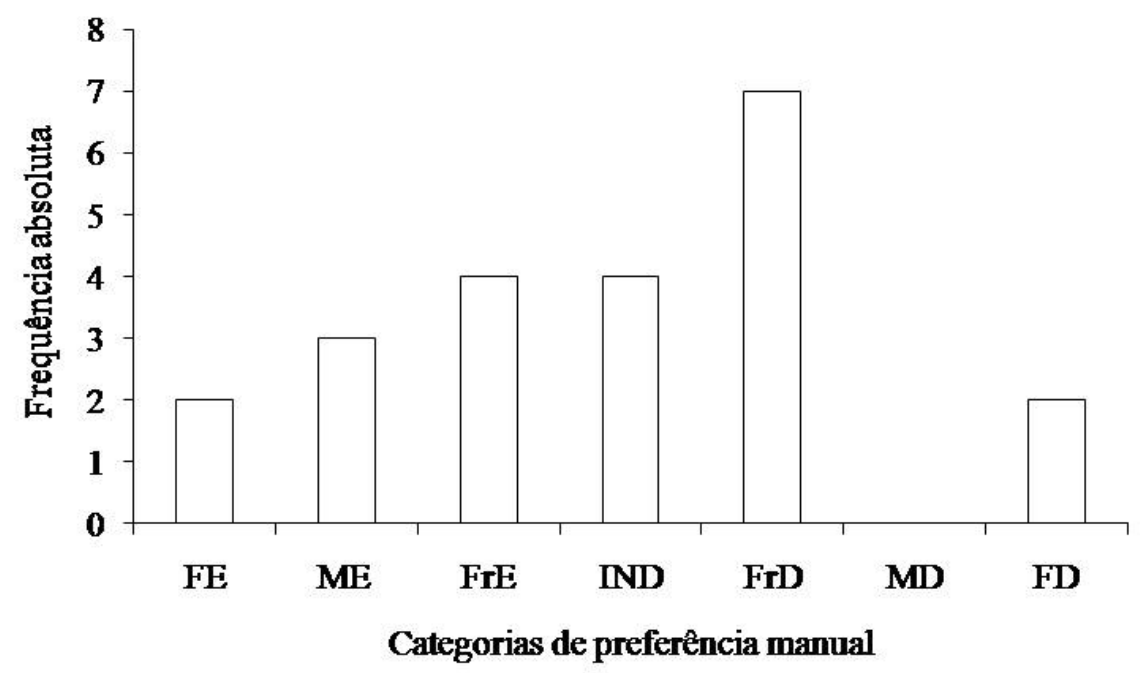

Figura 1. Distribuição de frequências absolutas da preferência manual no alcançar em 7 categorias.

Nota. Forte Preferência Manual Esquerda, FE; Moderada Esquerda, ME; Fraca Esquerda, FrE; Indefinido, IND; Fraca Direita, FrD; Moderada Direita, MD; e Forte Direita, FD.

Na Figura 2 é apresentada a distribuição de frequências de preferência manual para brinquedos posicionados à esquerda, na linha média e à direita. Nesta figura os bebês foram agrupados nas sete categorias de preferência manual descritas anteriormente. A análise descritiva destes dados revela que, quando o brinquedo era apresentado do lado esquerdo, a maioria dos bebês demonstrou preferência manual esquerda ou indefinida, com grande parte deles concentrados na categoria de forte preferência manual esquerda. Quando o brinquedo era apresenta- do do lado direito, a maioria dos bebês demonstrou preferência manual direita, sendo que a maior parte dos bebês se concentrou na categoria de fraca preferência manual direita. Para a posição central foi verificado um equilíbrio entre bebês com preferência manual direita e esquerda, com a maioria deles concentrados entre as categorias de preferência manual esquerda moderada e preferência manual direita fraca. Assim, estes dados indicam que os bebês apresentaram uma tendência geral em realizar movimentos de alcance ipsilaterais, modificando 
sua preferência manual de acordo com a posição do alvo. Algo interessante a ser observado é que parte dos bebês apresentou forte preferência manual, fazendo também alcances contralaterais. Esse comportamento foi detectado tanto para bebês com preferência manual direita $(n=3)$ quanto esquerda $(n=4)$.
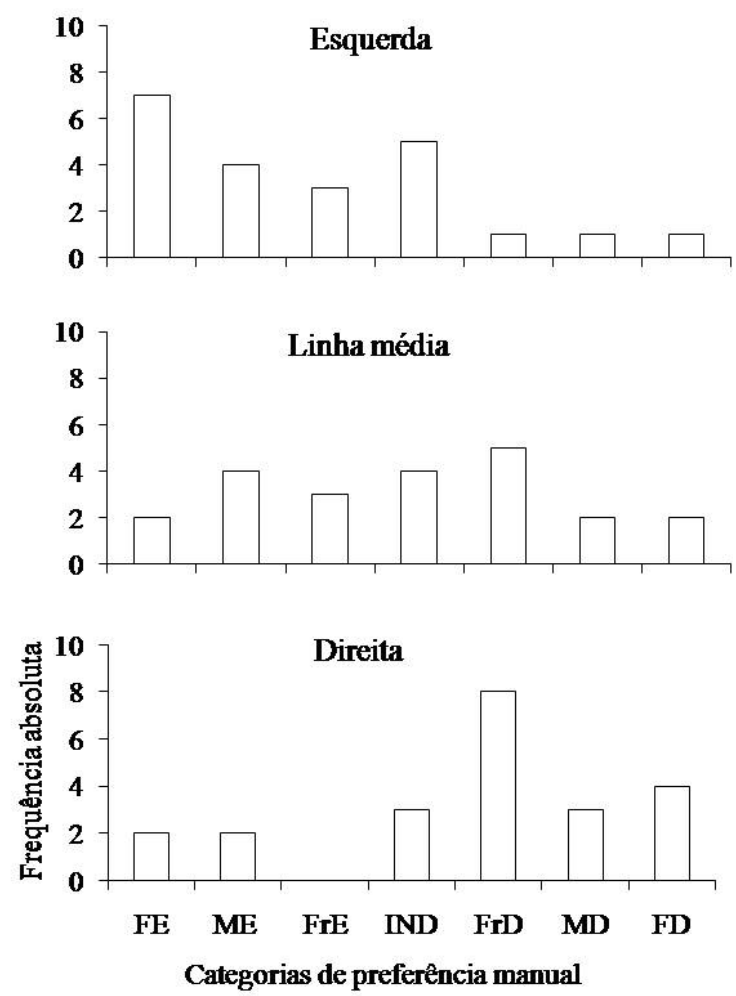

Figura 2. Distribuição de frequências absolutas de preferência manual em função da posição de apresentação do brinquedo
Na Figura 3 são apresentados os índices médios de preferência manual observados em função do sexo e da posição do brinquedo. A análise de variância destes dados indicou efeito significante para o fator posição, $F(2,40)$ $=23,67, P<0,0001$, enquanto que o fator sexo, $F(1,20)$ $=0,05$, e interação, $F(2,40)=0,99$, ficaram distantes de atingir valores significantes. $\mathrm{O}$ efeito significante do fator posição foi devido à detecção de diferenças em todas as comparações entre as três posições de alcance, com índices médios de $-0,39(D P=0,59),-0,05(D P=0,60) \mathrm{e}$ $0,26(D P=0,60)$, respectivamente para as posições esquerda, linha média e direita $\left(P_{\mathrm{s}}<0,005\right)$. Os resultados desta análise corroboram a análise descritiva, mostrando que a seleção do braço para alcance é influenciada pela posição de apresentação do brinquedo. A ausência de efeitos significantes relacionados ao fator sexo revela que este comportamento foi similar para bebês dos sexos masculino e feminino.

A análise dos resultados do Inventário de Dominância Lateral de Edimburgo revelou que as mães de todos os bebês possuíam preferência manual direita; dentre os pais, dois possuíam preferência indefinida, um possuía preferência esquerda e os demais preferência direita. Portanto, 13 bebês ( 9 com preferência esquerda e 4 com preferência indefinida) do grupo de 22 participantes demonstraram preferência manual incongruente com aquela manifestada pelas suas mães. Não foi encontrada congruência entre preferência manual esquerda ou indefinida dos pais com a preferência manual de seus bebês.

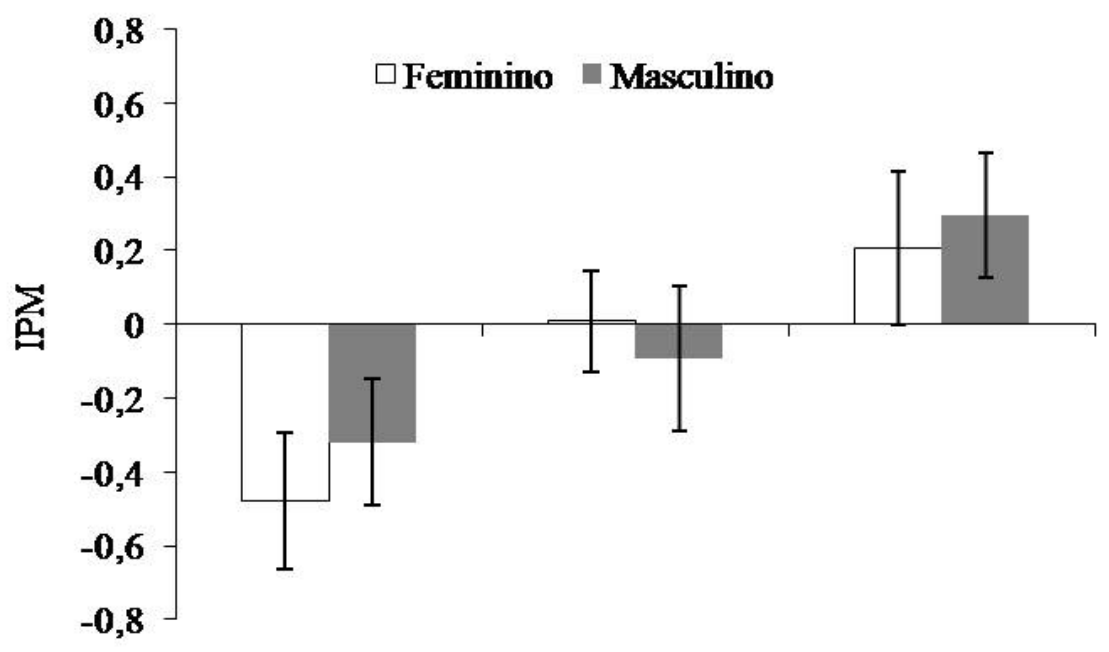

Esquerda Linha média Direita

Posição dos brinquedos

Figura 3. Índices médios de preferência manual (IPM) observados em função do sexo e da posição do brinquedo

Nota. Erro padrão representado por traços verticais. 


\section{Discussão}

O propósito do presente estudo foi analisar a direção e magnitude da preferência manual em bebês de 5 meses de idade em tarefas de alcançar objetos posicionados na linha média, à direita e à esquerda do corpo do bebê. Os resultados indicaram que a preferência manual é detectável em estágios iniciais do desenvolvimento motor. A análise individualizada revelou que aos 5 meses de idade já se observam preferências manuais bem definidas, incluindo casos de preferência manual consistente, caracterizada por alcances com o membro preferido no espaço contralateral. Uma característica em particular foi bastante distinta do padrão de manualidade de indivíduos adultos: a distribuição de preferências manuais entre os bebês. Os valores aqui apresentados indicaram frequências equivalentes entre preferência manual direita e esquerda nos bebês, sugerindo que neste período do desenvolvimento o viés populacional de preferência pelo lado direito ainda não está estabelecido.

Um dos principais achados originais deste estudo foi a distribuição dos bebês em categorias de preferência manual. Estudos prévios em bebês têm se concentrado principalmente na análise da frequência de movimentos de alcance e preensão com cada mão (Morange \& Bloch, 1996; Rönnqvist \& Domellöf, 2006), não oferecendo dados mais refinados sobre a preferência manual em períodos iniciais do desenvolvimento motor. A análise dos dados do presente estudo indicou que entre os bebês avaliados foram detectadas distintas categorias de preferência manual, assim como observado preliminarmente em indivíduos adultos (Peters \& Murphy, 1992). Um aspecto importante a ser observado é que foi encontrada a mesma incidência de bebês com forte preferência manual esquerda e forte preferência manual direita, o que demonstra que nesse estágio do desenvolvimento motor a tendência de preferência manual direita observada em indivíduos adultos não está estabelecida. Além disso, dentre os bebês que demonstraram preferência manual direita a maioria apresentou fraca preferência, enquanto que a maior parte dos bebês que demonstraram preferência manual esquerda foram classificados nas categorias de preferência fraca e moderada. Estes resultados mostram que na ação de alcançar alvos estáticos há uma variedade de categorias de preferência manual em bebês, com distribuição ainda mais diversificada do que tem sido observado em indivíduos adultos.

Apesar de resultados de estudos prévios em bebês (Sacco et al., 2006; Strogonova et al., 2004) e crianças (Teixeira \& Gasparetto, 2002) indicarem diferenças no comportamento lateralizado em função do sexo, no presente estudo essas diferenças não foram encontradas. Um aspecto metodológico que pode estar envolvido nessa diferença de resultados é o fato de os estudos de Sacco et al. (2006) e Strogonova et al. (2004) terem avaliado bebês ao final do primeiro ano de vida, enquanto que em nosso estudo foram avaliados bebês ainda no primeiro semestre de vida. Os resultados obtidos no presente estudo estão em consonância com achados de estudos em que foram avaliados neonatos (Liederman \& Kinsbourne, 1980) e bebês ainda no primeiro semestre de vida (Caplan \& Kinsbourne, 1976; Petrie \& Peters, 1980). Assim, caso haja uma diferença na preferência manual relacionada ao sexo, é provável que ela se desenvolva em um período posterior à idade de 5 meses.

Uma observação de interesse para compreender a lateralização do comportamento humano foi que a preferência manual direita foi menor do que aquela frequentemente observada na população adulta. Como tanto durante a infância, quanto durante a vida adulta - da juventude à velhice - tem sido identificado que a incidência de indivíduos com preferência manual direita tende a aumentar (cf. Ashton, 1982; Hinojosa, Sheu, \& Michel, 2003), supõe-se que com o avanço da idade os indivíduos se tornem mais lateralizados (Singh et al., 2001; Teixeira, 2008). Assim, se a expectativa de distribuição populacional de aproximadamente $90 \%$ de indivíduos destros (Brackenridge, 1981; Porac et al., 1980) for confirmada, aproximadamente $80 \%$ dos bebês com preferência manual esquerda ou indefinida deverão mudar sua preferência manual para o lado direito em idades mais avançadas. Com base nesta observação, aparentemente não há uma relação direta entre a preferência manual em fases iniciais da infância e aquela observada na população adulta, como tem sido sugerido a partir de resultados de estudos prévios (Coryell, 1985; Hepper, Wells, \& Lynch, 2005; Hinojosa et al., 2003; Michel \& Harkins, 1986; Michel, Sheu, \& Brumley, 2002). Os resultados aqui apresentados, portanto, são inconsistentes com a proposição de que a preferência manual é definida predominantemente por fatores genéticos (Annett, 1978). Caso houvesse um gene definindo a lateralidade, com viés de lateralização favorável ao lado direito do corpo, a maior proporção de preferência lateral direita deveria se manifestar logo nos primeiros meses de vida. Nesse sentido, uma proposta conciliatória entre as visões de determinação da preferência manual por fatores genéticos e por fatores ambientais é de que predisposições inatas, representadas por tendências laterais precoces, sejam reforçadas ou modificadas pela contínua interação com o ambiente durante o ciclo de vida de um indivíduo (Souza \& Teixeira, 2011). Por esta perspectiva, assimetrias estruturais entre os dois hemisférios cerebrais, determinadas geneticamente, seriam apenas um dos elementos com potencial de enviesar a lateralização do comportamento motor à direita ou à esquerda.

Como hipotetizado nesta investigação, a posição do brinquedo influenciou a escolha da mão para realizar o alcance. Em concordância com estudos prévios em bebês (Morange \& Bloch, 1996), crianças (Gabbard \& Helbig, 2004; Hill \& Khanen, 2009; Leconte \& Fagard, 2006) e adultos (Bryden \& Roy, 2006), as posições laterais do brinquedo induziram a realização de alcances ipsilaterais na maioria dos bebês. Resultados de um estudo recente 
indicaram que a escolha de uma das mãos para alcançar objetos localizados lateralmente é influenciada pela dominância motora e pela demanda da tarefa (Gabbard \& Helbig, 2004). Alcances na linha média e ipsilaterais à mão dominante seriam predominantemente influenciados pela dominância motora, sendo realizados com a mão preferida. Em alcances contralaterais, por outro lado, o fator predominante seria a proximidade do objeto. Alcançar um objeto com a mão que está mais próxima é mais eficiente do ponto de vista biomecânico (Carey, Hargreaves, $\&$ Goodale, 1996), o que parece ser um importante elemento indutor da preferência manual quando se considera a posição do alvo para alcance com referência a coordenadas egocêntricas. A flutuação da preferência manual em função da localização espacial do alvo revela, assim, que a disposição de objetos no ambiente é um elemento modulador em potencial da preferência manual.

Estudos em animais têm sugerido que o treino específico em uma tarefa manual em ambiente assimetrizado é capaz de estabelecer a preferência lateral, assim como reverter preferências laterais preexistentes (McGonigle \& Flook, 1978; Warren, 1958). Isto é, a indução contínua de uso de um membro, através da disposição de objetos em local que possibilitava apenas o alcance com este membro, foi evidenciada levar ao estabelecimento de preferência lateral pelo membro treinado para a tarefa praticada e para outras tarefas relacionadas (McGonigle \& Flook, 1978). A partir dessa perspectiva, poderia ser hipotetizado que o oferecimento sistemático de objetos em um dos lados do espaço de trabalho do bebê tem potencial de induzir a realização de movimentos mais frequentes com sua mão ipsilateral. Isto é, uma mãe ou cuidador, por exemplo, que ofereçam frequentemente os brinquedos do lado direito do bebê induziriam o uso mais frequente da mão direita para alcance e preensão. Visto que a mudança de preferência manual em função de prática unilateral tem sido mostrada ser generalizável para tarefas relacionadas àquela praticada (Teixeira \& Okazaki, 2007), a prática lateralizada em tarefas de alcance em fases iniciais do desenvolvimento motor poderia desempenhar o papel de difusor da preferência manual para tarefas variadas de preensão e manipulação.

Um aspecto adicional a ser considerado nos resultados do presente estudo é o fato de não ter sido observada congruência entre a preferência manual esquerda de vários bebês e a preferência manual materna, como observado em estudos prévios (Harkins \& Michel, 1988; Harkins \& Uzgiris, 1991). Uma das conclusões a que este resultado conduz é que aparentemente o comportamento lateral dos bebês não foi afetado por um componente genético. Para explicar a incidência elevada de preferência manual esquerda, como revelado pelos resultados, seria necessário haver uma distribuição atípica de preferências laterais entre mães/pais. Ainda que a ausência de um gene determinante da dominância lateral, como teorizado por Annett (1978), pudesse levar à preferência manual direita, a presença de apenas um caso de preferência esquerda entre os pais indica um perfil de lateralização com claro viés à direita. Embora a manualidade da mãe seja um elemento potencialmente importante no desenvolvimento da lateralidade infantil, por efeito de comportamento imitativo dos bebês (cf. Fagard \& Lemoine, 2006; Nagy et al., 2005), os dados aqui apresentados sugerem que outros aspectos possuem maior peso no estabelecimento da preferência manual em estágios iniciais do desenvolvimento motor. Em consonância com o efeito de alcances ipsilaterais aqui observado, a disposição espacial de brinquedos no espaço de permanência do bebê poderia ser um aspecto importante da lateralização do comportamento manual, assim como outros elementos que tornem o ambiente cotidiano do bebê assimétrico.

\section{Referências}

Annett, M. (1970). The growth of manual preference and speed. British Journal of Psychology, 61(4), 545-558.

Annett, M. (1978). Genetic and nongenetic influences on handedness. Behavior Genetics, 8(3), 227-249.

Annett, M. (1999). Left-handedness as a function of sex, maternal versus paternal inheritance, and report bias. Behavior Genetics, 29(2), 103-114.

Ashton, G. C. (1982). Handedness: An alternative hypothesis. Behavior Genetics, 12(2), 125-147.

Barros, R. M. L., Brenzikofer, R., Leite, N. J., \& Figueroa, P. J. (1999). Desenvolvimento e avaliação de um sistema para análise cinemática tridimensional de movimentos humanos. Revista Brasileira de Engenharia Biomédica, 15(1-2), 79-86.

Brackenridge, C. (1981). Secular variation in handedness over ninety years. Neuropsychologia, 19(3), 459-462.

Bryden, P. M., \& Roy, E. A. (2006). Preferential reaching across regions of hemispace in adults and children. Developmental Psychobiology, 48(2), 121-132.

Caplan, P. I., \& Kinsbourne, M. (1976). Baby drops the rattle: Asymmetry of duration of grasp by infants. Child Development, 47(2), 532-534.

Carey, D. P., Hargreaves, E. L., \& Goodale, M. A. (1996). Reaching to ipsilateral or contralateral targets: Withinhemisphere visuomotor processing cannot explain hemispatial differences in motor control. Experimental Brain Research, 112(3), 496-504.

Corbetta, D., \& Thelen, E. (1996). The developmental origins of bimanual coordination: A dynamic perspective. Journal of Experimental Psychology: Human Perception and Performance, 22(2), 502-522.

Corbetta, D., \& Thelen, E. (1999). Lateral biases and fluctuations in infants' spontaneous arm movements and reaching. Developmental Psychobiology, 34(4), 237-255.

Corbetta, D., Williams, J., \& Snapp-Childs, W. (2006). Plasticity in the development of handedness: Evidence from normal development and early asymmetric brain injury. Developmental Psychobiology, 48(6), 460-471.

Coryell, J. (1985). Infant rightward asymmetries predict righthandedness in childhood. Neuropsychologia, 23(2), 269-271.

Fagard, J. (2000). Linked proximal and distal changes in the reaching behavior of 5-to 12-month-old human infants grasping objects of different sizes. Infant Behavior and Development, 23(3-4), 317-329. 
Fagard, J., \& Lemoine, C. (2006). The role of imitation in the stabilization of handedness during infancy. Journal of Integrative Neuroscience, 5(4), 519-533.

Gabbard, C., \& Helbig, C. R. (2004). What drives children's limb selection for reaching in hemispace? Experimental Brain Research, 156(3), 325-332.

Gesell, A., \& Ames, L. B. (1947). The development of handedness. Journal of Genetic Psychology, 70, 155-175.

Harkins, D. A., \& Michel, G. F. (1988). Evidence for a maternal effect on infant hand-use preferences. Developmental Psychobiology, 21(6), 535-541.

Harkins, D. A., \& Uzgiris, I. C. (1991). Hand-use matching between mothers and infants during the first year. Infant Behavior and Development, 14, 289-298.

Hepper, P. G., McCartney, G. R., \& Shannon, E. A. (1998). Lateralised behaviour in first trimester human foetuses. Neuropsychologia, 36(6), 531-534.

Hepper, P. G., Shahidullah, S., \& White, R. (1991). Handedness in the human fetus. Neuropsychologia, 29(11), 1107-1111.

Hepper, P. G., Wells, D. L., \& Lynch, C. (2005). Prenatal thumb sucking is related to postnatal handedness. Neuropsychologia, 43(3), 313-315.

Hill, E. L., \& Khanem, F. (2009). The development of hand preference in children: The effect of task demands and links with manual dexterity. Brain and Cognition, 71(2), 99-107.

Hinojosa, T., Sheu, C. F., \& Michel, G. F. (2003). Infant handuse preferences for grasping objects contributes to the development of a hand-use preference for manipulating objects. Developmental Psychobiology, 43(4), 328-334.

Hopkins, B., \& Rönnqvist, L. (2002). Facilitating postural control: Effects on the reaching behavior of 6-month-old infants. Developmental Psychobiology, 40(2), 168-182.

Leconte, P., \& Fagard, J. (2006). Which factors affect hand selection in children's grasping in hemispace? Combined effects of task demand and motor dominance. Brain and Cognition, 60(1), 88-93.

Liederman, J., \& Kinsbourne, M. (1980). The mechanism of neonatal rightward turning bias: A sensory or motor asymmetry? Infant Behavior and Development, 3, 223-238.

McGonigle, B. O., \& Flook, J. (1978). The learning of hand preferences by squirrel monkey. Psychological Research, 40(1), 93-98.

Meng, L. (2007). The rate of handedness conversion and related factors in left-handed children. Laterality, 12(2), 131-138.

Michel, G. E., \& Harkins, D. A. (1986). Postural and lateral asymmetries in the ontogeny of handedness during infancy. Developmental Psychobiology, 19(3), 247-258.

Michel, G. F., Sheu, C., \& Brumley, M. R. (2002). Evidence of a right-shift factor affecting infant hand-use preferences from 7 to 11 months of age as revealed by latent class analysis. Developmental Psychobiology, 40(1), 1-13.

Morange, F., \& Bloch, H. (1996). Lateralization of the approach movement and the prehension movement in infants from 4 to 7 months. Early Development and Parenting, 5, 81-92.

Nagy, E., Compagne, H., Orvos, H., Pal, A., Molnar, P., Janszky, I., et al. (2005). Index finger movement imitation by human neonates: Motivation, learning, and left-hand preference. Pediatric Research, 58(4), 749-753.

Oldfield, R. C. (1971). The assessment and analysis of handedness: The Edinburgh Inventory. Neuropsychologia, 9(1), 97-113.

Peters, M., \& Murphy, K. (1992). Cluster analysis reveals at least three, and possibly five distinct handedness groups. Neuropsychologia, 30(4), 373-380.
Petrie, B. F., \& Peters, M. (1980). Handedness: Left/right differences in intensity of grasp response and duration of rattle holding in infants. Infant Behavior and Development, 3, 215221.

Porac, C., Coren, S., \& Duncan, P. (1980). Life-span age trends in laterality. Journal of Gerontology, 35(5), 715-721.

Ramsay, D. S. (1980). Onset of unimanual handedness in infants. Infant Behavior \& Development, 3, 377-385.

Rocha, N. A. C. F., Silva, F. P. S., \& Tudella, E. (2006). Influência do tamanho e da rigidez dos objetos nos ajustes proximais e distais do alcance de lactentes. Revista Brasileira de Fisioterapia, 10(3), 263-269.

Rönnqvist, L., \& Domellöf, E. (2006). Quantitative assessment of right and left reaching movements in infants: A longitudinal study from 6 to 36 months. Developmental Psychobiology, 48(6), 444-459.

Sacco, S., Moutard, M., \& Fagard, J. (2006). Agenesis of the corpus callosum and the establishment of handedness. Developmental Psychobiology, 48(6), 472-481.

Singh, M., Manjary, M., \& Dellatolas, G. (2001). Lateral preferences among Indian school children. Cortex, 37(2), 231-241.

Souza, R. M., \& Teixeira, L. A. (2011). Sobre a relação entre filogenia e ontogenia no desenvolvimento da lateralidade na infância. Psicologia: Reflexão e Crítica, 24(1), 60-70.

Strogonova, T. A., Pushina, N. P., Orekhova, E. V., Posikera, I. N., \& Tsetlin, M. M. (2004). Functional brain asymmetry and individual differences in hand preference in early ontogeny. Human Physiology, 30(1), 14-23.

Teixeira, L. A. (2008). Categories of manual asymmetry and their variation with advancing age. Cortex, 44(6), 707-716.

Teixeira, L. A., \& Gasparetto, E. R. (2002). Lateral asymmetries in the development of the overarm throw. Journal of Motor Behavior, 34(2), 151-160.

Teixeira, L. A., \& Okazaki, V. H. A. (2007). Shift of manual preference by lateralized practice generalizes to related motor tasks. Experimental Brain Research, 183(3), 417-423.

Thelen, E., Corbetta, D., \& Spencer, J. P. (1996). Development of reaching during the first year: Role of movement speed. Journal of Experimental Psychology: Human Perception and Performance, 22(5), 1059-1076.

Von Hofsten, C. (1982). Eye-hand coordination in the newborn. Developmental Psychology, 18(3), 450-461.

Warren, J. M. (1958). The development of paw preference in cats and monkeys. The Journal of Genetic Psychology, 93(2), 229-236.

Zverev, Y. P. (2006). Cultural and environmental pressure against left-hand preference in urban and semi-urban Malawi. Brain and Cognition, 60(3), 295-303. 